\title{
Body Mass Index (BMI) is not a cancer risk factor for BRCA1/2 carriers: a Systematic Review and Meta-analysis of case-control studies
}

\section{Mario Giuseppe Mirisola}

University of Palermo: Universita degli Studi di Palermo

\section{Antonio Galvano}

University of Palermo: Universita degli Studi di Palermo

\section{Valerio Gristina}

University of Palermo: Universita degli Studi di Palermo

\section{Maria La Mantia}

University of Palermo: Universita degli Studi di Palermo

\section{Sofia Cutaia}

University of Palermo: Universita degli Studi di Palermo

\section{Valentina Calò}

University of Palermo: Universita degli Studi di Palermo

\section{Nadia Barraco}

University of Palermo: Universita degli Studi di Palermo

\section{Marco Bono}

University of Palermo: Universita degli Studi di Palermo

\section{Marta Castiglia}

University of Palermo: Universita degli Studi di Palermo

Alessandro Perez

University of Palermo: Universita degli Studi di Palermo

\section{Stefania Cusenza}

University of Palermo: Universita degli Studi di Palermo

\section{Lavinia Insalaco}

University of Palermo: Universita degli Studi di Palermo

\section{Luisa Castellana}

University of Palermo: Universita degli Studi di Palermo

\section{Fabio Fulfaro}

University of Palermo: Universita degli Studi di Palermo

\section{Antonio Russo ( $\square$ antonio.russo@usa.net)}

Università degli Studi di Palermo: Universita degli Studi di Palermo https://orcid.org/0000-0002-4370- 


\section{Viviana Bazan}

University of Palermo: Universita degli Studi di Palermo

\section{Methodology}

Keywords: BMI, Meta-Analysis, Cancer Risk, systematic review, BRCA1/2

Posted Date: March 19th, 2021

DOI: https://doi.org/10.21203/rs.3.rs-337397/v1

License: (c) (1) This work is licensed under a Creative Commons Attribution 4.0 International License. Read Full License 


\section{Abstract \\ Background}

Breast cancer susceptibility gene 1 and 2 (BRCA 1/2) pathogenic germline variants (gPV) are involved in an increased cumulative risk for cancers (above all breast and ovarian cancers). Overweight/obesity is a well-known systemic condition conferring higher cancer risk too.

\section{Methods}

We performed a systematic review collecting data on Medline, Scopus, and Cochrane-Library database until August 2020. We included four case-control studies (Fu et al, Bissonauth et al, Khachatryan et al, Nkondjoc et al) assessing the risk for cancer according to different BMI strata in BRCA-positive healthy individuals.

\section{Results}

Four studies for a total of 1148 patients evaluated breast and ovarian cancer risk in a healthy BRCA1/2 population. No other tumor histotypes risk has been observed within the selected population. Pooled results demonstrated that different BMI conditions (lower or upper $25 \mathrm{~kg} / \mathrm{m}^{2}$ ) were not associated with increased cancer risk (OR 1.15, 95\% $\mathrm{Cl} 0.92-1.44$ and OR $1.48,95 \% \mathrm{Cl} 0.84-2.62$ respectively). Additionally, no differences were reported according to menopausal status.

\section{Conclusion}

Despite the need for other prospective investigations in larger cohorts, our results suggest no BMI contribution in cancer risk in this special population, determining a new important point of view and a new potential field of investigation.

\section{Background}

Breast cancer susceptibility gene 1 and 2 (BRCA 1/2) are tumor suppressor genes with essential roles in genome integrity maintenance (1). Alterations in BRCA1 or BRCA2 genes predispose to different types of cancer though they are mostly associated with hereditary breast-ovarian cancer syndromes (HBOC) (2). Their gene products play different but equally essential roles in homologous recombination (HR), a highly accurate DNA repair mechanism that takes advantage of undamaged sister chromatid to repair replication-associated DNA double-strand breaks (DSBs). Other gene products such as PALB2, RAD51C, RAD51D, BRIP, CHEK2 take part in HR and cancer-associated mutations have been described in these genes as well (3-5). 
The BRCA1/2 pathogenic germline gene variants (gPVs) are inherited in an autosomal-dominant manner. The incomplete penetrance observed in a pedigree chart is thought to depend on gender, environment, and the existence of modifier loci (6-9). In women with gPV in BRCA 1 or BRCA 2 genes, the average cumulative risk of developing breast cancer $(\mathrm{BC})$ by 80 years of age is $72 \%$ and $69 \%$ respectively (10) with an increased risk of contralateral breast cancer. On the contrary, the hereditary ovarian cancer (OC) risk, mainly for epithelial histotypes, is $44 \%$ for BRCA1 gPV carriers and $17 \%$ for BRCA2 gPV carriers (11). In males, gPV increases the risk of breast, prostate, and pancreatic cancer, albeit to a much lesser extent (12).

The obesity pandemic increases the risk for multiple serious conditions and is regarded as a cause of preventable cancer risk. Overweight/obesity is recognized as a key environmental risk factor for several cancer types. Excessive weight causes $20 \%$ of all cancers (13), while postmenopausal women show an association with obesity in $50 \%$ of all cancers (14). However, while epidemiological studies confirm a direct association between overweight and cancer, the role of obesity alone as a cancer risk factor in all tissues is controversial $(15,16)$. Besides, further analysis has suggested the obesity-cancer bond is sexspecific in a wide range of different malignancies and for various geographic populations (17). On the contrary, different ethnic groups show different susceptibility. For example, African Americans are more susceptible to cancer than Hispanics who are more protected. Interestingly, the association of increased $\mathrm{BMI}$ with breast cancer is particularly strong in Asia-Pacific populations suggesting the existence of genetic and or lifestyle differences capable to modulate the effect(18).

Besides, multiple mechanisms explain the association between cancer and obesity status since adipose tissue is an important endocrine organ(19). In addition, obesity and in general excessive abdominal fat induces a chronic inflammatory status conferring additional cancer risk beyond obesity itself (19-21). It is known that abdominal fat-dependent inflammation and cellular oxidative stress can result in DNA damage and overwhelm or reduce the repair capacity and/or accuracy of various DNA repair mechanisms, including HR, driving the accumulation of cancer-associated mutations over time (22). Moreover, model organisms research has demonstrated that over-activation of major nutrient responsive pathways, namely PI3K/PKA/TOR resulting in decreased genome stability.

Several cancer prevention programs including the case of BRCA gPV carriers emphasize the role of a "healthy lifestyle" always stressing the negative effect of alcohol consumption, western-style nutrition, and physical inactivity because of their role in increasing cancer risk. However, the increasing need for precision medicine requires to validate these general lifestyle recommendations in different genetic backgrounds. Hence, we made a systematic review and meta-analysis of body mass index (BMI) in a cohort of BRCA1/2 mutation carriers to eventually evaluate the additional effect of BMI as a cancer risk in BRCA selected healthy individuals.

\section{Materials And Methods}

\section{Search Strategy and Study Selection}


We searched for the results of prospective or retrospective trials comparing BRCA $1 / 2$ carriers for cancer risk. Moreover, we excluded trials whose results for cancer risk were not available, trials with small accrual (less than 10 patients), and ongoing studies. Studies have been included if BRCA $1 / 2$ carriers with $\mathrm{BMI}$ evaluation available for the analysis. $\mathrm{BMI}$ is the main parameter to evaluate and determine the degree of obesity (23).

$\mathrm{BMI}$ is a calculated value (body weight $[\mathrm{Kg}]$ divided by square height $\left[\mathrm{m}^{2}\right]$ ), stratifying individuals into different classes: underweight $(\mathrm{BMI}<18.5)$ or normal weight $(18.5-24.99)$ versus overweight $(25-29.99)$ or obese $(>30)(24)$.

The research was carried out using specific Mesh terms such as "BMI" and "neoplasms" and free text terms such as "cancer risk" or "cancer" and "body size" using Boolean operators (Figure S1). Data collected on Medline (PubMed), Scopus, and Cochrane-Library database were collected until August 2020, limiting the search to English-only articles; other potential abstracts published on American Society of Clinical Oncology (ASCO) and the European Society of Medical Oncology (ESMO) databases were also collected. The National Institute of Health $(\mathrm{NIH})$ website (www.clinicaltrials.gov) was also explored to select results from not yet published ongoing studies as a source of gray literature. Our research protocol was previously registered on PROSPERO 2020 database with the following code: CRD42020203910. The outcomes of our analysis were the cancer risk defined as the proportion of BRCA1/2 carriers developing cancer during their life, according to BMI subgroup (normal: up to $25 \mathrm{~kg} / \mathrm{m}^{2}$; overweight/obese:> 25 $\mathrm{kg} / \mathrm{m}^{2}$ ) or menopausal status. Only data on BRCA1/2 carriers aged $\geq 18$ years, with no sex restrictions were included in our analysis. The trial selection was performed independently by two authors (A.G. and V.G.) who screened and identified possible relevant articles according to inclusion criteria. After trial selection, authors evaluated outcomes relevance and consequently included their results in the final analysis. Disagreements were discussed and solved by consulting a third author (M.G.M.). Finally, four case-control articles were included in our final analysis (Table 1).

Table 1. Characteristics of the included studies. OR: Odd Ratio; $\mathrm{n}$. : sample size; BMl: Body Mass Index; Cl: Confidence Interval; PRE: pre-menopausal Status; POST: post-menopausal Status; NA: not available. 


\begin{tabular}{|c|c|c|c|c|c|c|}
\hline Reference & $\frac{\text { Case }}{\text { (n.). }}$ & $\frac{\text { Controls }}{\text { (n. })}$ & $\begin{array}{l}\text { Age } \\
\text { (Mean) }\end{array}$ & $\begin{array}{l}\text { OR } \\
\frac{\text { BMI } £ 25}{\text { and }>25} \\
(95 \% \mathrm{Cl}) .\end{array}$ & $\begin{array}{l}\text { OR } \\
\text { Menopausal } \\
\text { Status normal } \\
\text { BMI } \\
(\underline{95 \% \mathrm{Cl}) .}\end{array}$ & $\begin{array}{l}\text { OR } \\
\text { Menopausal Status } \\
\underline{\text { overweightlobese }} \\
\underline{\text { BMI }} \\
(\underline{95 \% \mathrm{Cl}) .}\end{array}$ \\
\hline $\begin{array}{l}\text { Fu } \\
\text { et al } \\
2015(25)\end{array}$ & 62 & 57 & 50.27 & $\begin{array}{l}\text { BMI £ } 25 \\
1.18 \\
(1.14- \\
1.74)\end{array}$ & N.A. & N.A. \\
\hline $\begin{array}{l}\text { Bissonauth } \\
\text { et al } \\
2009(26)\end{array}$ & 280 & 280 & 51.0 & $\begin{array}{l}\text { BMI } £ 25 \\
1.25 \\
(0.76- \\
1.85) \\
\text { BMI > } 25 \\
1.55 \\
(0.77- \\
1.89)\end{array}$ & $\begin{array}{l}\text { PRE } \\
1.37(0.96-2.60) \\
\text { POST } \\
1.65(0.96-2.70)\end{array}$ & $\begin{array}{l}\text { PRE } \\
1.02(0.52-2.07) \\
\text { POST } \\
1.19(0.92-2.01)\end{array}$ \\
\hline $\begin{array}{l}\text { Khachatryan } \\
\text { et al } \\
2011(27)\end{array}$ & 150 & 152 & 55.79 & $\begin{array}{l}\text { BMI } £ \\
\mathbf{2 5} \\
1.29 \\
(0.68- \\
2.44) \\
\text { BMI > } 25 \\
2.42 \\
(1.24- \\
4.75)\end{array}$ & N.A. & N.A. \\
\hline $\begin{array}{l}\text { Nkondjock } \\
\text { et al } \\
2006(28)\end{array}$ & 89 & 48 & 56.2 & $\begin{array}{l}\text { BMl } f \\
25 \\
\\
0.58 \\
(0.23- \\
1.48) \\
\\
\text { BMI > } 25 \\
\\
0.72 \\
(0.30- \\
1.73)\end{array}$ & N.A. & N.A. \\
\hline
\end{tabular}

Data Extraction and Assessment of the Quality of the Included Studies 
Raw data were collected independently by two authors (A.G. and V.G.) and the disagreements were solved by consulting a third author (M. G. M.). Data were collected in a predefined file in which we included: Reference, study population, age (mean or median). BRCA1/2 cohorts, BMI (mean), OR according to BMI predefined subgroups ( $\leq 25$ or $>25$ ). If the same article was published with different follow-up over time, we decided to select the most updated. According to these criteria, only four studies (Bissonauth et al (26), Fu et al (25), Nkondjoc et al (28), Khachatryan et al (27)) contained data on BMI and cancer risk (breast cancer above all) and one study (Bissonauth et al(26)) on the premenopausal status and were included in the final analysis. The quality of the included studies was evaluated through the NewcastleOttawa Quality Assessment Scale for cohort and case-control studies. A quality score for observational studies was calculated based on the three major components of this scale and in particular: (1) selection of the groups included in the study, (2) comparability, (3) assessment of the outcome of exposure. Studies were classified according to the sum of these three areas up to a total of 9 points, representing the highest methodological quality (29).

\section{Statistical Analysis}

Statistical analysis was performed using RevMan ver 5.3, and Comprehensive Meta-analysis version 2.2.064 (30). The outcome selected to perform the meta-analysis was the cancer risk, with the relative $95 \%$ confidence intervals ( $\mathrm{Cl}$ ). Odds ratios (ORs) were used as an association measure for cancer risk, considered as the ratio between the number of events on non-events. For instance, if the odds ratio $>1$, no cancer risk would be observed according to BMI subgroups; conversely, a greater risk would be present if the odds ratio $<1$. Heterogeneity between studies was assessed using the Cochrane $Q$ test and the Isquared test. If I-squared was greater than $50 \%$, it was associated with a high risk of heterogeneity and then the meta-analysis was computed using the random effect-based model by Der Simonian and Laird; otherwise, the meta-analysis was computed through the fixed effect-base model according to MantelHaenszel (31). Furthermore, publication bias risk was explored through Egger's test producing the related funnel plot for asymmetry. The manuscript was drafted following the Preferred Reporting Items for Systematic Reviews and Meta-Analyzes (PRISMA) guidelines (Figure S2) (32). The p values were considered significant if $p \leq 0.05$.

\section{Results}

\section{Association between BRCA and BMI}

The literature search identified a total of 74 records; 14 records were excluded because of duplicates while 4 records were finally ruled out because of reviews, letters, commentaries, editorials, or protocols. A total of 56 records were assessed for eligibility while 52 were excluded because no drugs of interest or data about the principal outcomes of our indirect comparison were reported. Finally, a total of four studies met our inclusion/exclusion criteria and were included within the final analysis; four studies for a total of 1148 patients evaluated breast and ovarian cancer risk in a healthy BRCA1/2 population (Figure 1). Intriguingly, no other tumor histotypes risk has been observed within the selected population. 
Specifically, one of these trials (Bissonauth et al) provided its result stratified according to menopausal status (pre- or post-).

Our pooled results demonstrated how different BMI conditions (lower or upper $25 \mathrm{~kg} / \mathrm{m}^{2}$ ) were not associated with increased cancer risk (OR 1.15, 95\% Cl $0.92-1.44$ and OR 1.48, 95\% Cl $0.84-2.62$ respectively) (Figure 2). Interestingly, obesity seemed to be not associated with higher chances of conferring cancer risk in such patients, whereas on the contrary presenting with a strong trend for statistical significance and therefore resulting to be a protective factor against breast-ovarian cancer risk in BRCA-positive healthy individuals. Similar results were obtained when BMI was investigated according to the menopausal status, confirming the lack of association with cancer risk (premenopausal, OR 1.24, $95 \% \mathrm{Cl} 0.83-1.85$; post-menopausal OR 1.34, 95\% Cl $0.98-1.83$ ) (Figure 3). The quality assessment reported a high overall quality for all included studies in all the three domains (selection, comparability, and outcome exposure; Table 2). Furthermore, no additional risk for potential publication bias was observed, not eventually affecting the final analysis (Figure 4).

Table 2. Summary of critical appraisal of included studies using the Newcastle-Ottawa Quality Assessment Scale (29).

\begin{tabular}{|c|c|c|c|c|}
\hline Study ID & $\begin{array}{l}\text { Selection } \\
\text { (max } 4 \text { stars) }\end{array}$ & $\begin{array}{l}\text { Comparability } \\
\text { (max } 2 \text { stars) }\end{array}$ & $\begin{array}{l}\text { Outcome or exposure } \\
\text { (max } 3 \text { stars) }\end{array}$ & Total \\
\hline Bissonauth et al(26) & 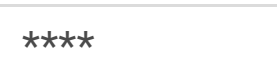 & ** & 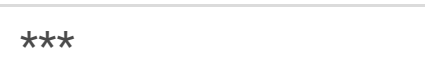 & 9 \\
\hline Fu et al(25) & 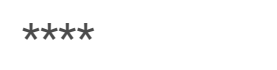 & $-*$ & $\star * *$ & 8 \\
\hline Nkondjock et al(28) & 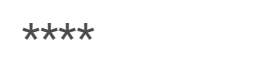 & $-*$ & $\star * *$ & 8 \\
\hline Khachatryan et al(27) & $\star \star \star \star \star ~$ & $-\star *$ & $\star \star *$ & 8 \\
\hline
\end{tabular}

\section{Selection}

1. Representativeness of intervention cohort: a) Truly representative of average BRCA $1 / 2$ carriers*; b) somewhat representative of average BRCA $1 / 2$ carriers*; c) only selected group of patients; no description of the derivation of cohort

2. Selection of non-intervention cohort: a) drawn from the same community as intervention cohort*; b) drawn from a different source; c) no description of the derivation of the non-intervention cohort

3. Ascertainment of intervention: a) health record*; b) structured interview*; c)written self-record; d) no description

4. Demonstration that outcome was not present t start of the study: a)yes*;b)no

\section{Comparability}


1. Comparability of cohorts on basis of design or analysis: a) study controls for age, sex, or frequency of sex*; b) study controls for any additional factors*

\section{Outcome}

1. Assessment of outcome: a) independent blind assessment*; b) record linkage*; c) self-report; d) no description

2. Was follow up long enough for outcomes to occur: a) yes (median duration of follow up> 6 months)*; b) no

3. Adequacy of follow up of cohort: a) complete follow up*; b) minimal loss of follow up ( $\leq 20 \%)$; c) follow up rate $<80 \%$ and no description of losses to follow up; no statement

\section{Discussion}

The last decade has seen the obesity-cancer link receiving increasing attention and epidemiological studies have confirmed that obesity is associated with a higher risk of several cancers, including colon, breast, endometrium, liver, kidney, esophagus, gastric, pancreatic, gallbladder, and leukemia (33). In particular, one of the well-established breast cancer risk factors in post-menopausal women is obesity status, which has also been linked to breast cancer recurrence and poorer survival in pre- and postmenopausal breast cancer (34). A meta-analysis on BMI and breast cancer risk for post-menopausal women confirmed the association and also found a "dose-dependent" association between BMI and cancer risk with a relative risk (RR) of $1.02 ; 1.12$, and 1.26 for people with a BMI of 25,30 and 35 respectively (35). Despite this clear obesity/cancer association the biological mechanisms responsible for this relationship are still controversial, as previously mentioned $(22,36)$. The purpose of the current study was to deeply investigate the causal relationship between BMI and the risk of developing cancer in BRCA selected healthy individuals. Namely, the literature search did not match tumors other than breast and/or ovarian histotype in this setting.

In the present article, we explored the hypothesis that the combination of BRCA $1 \backslash 2$ mutational status and elevated BMI might be not associated with an increased risk of breast cancer. Our meta-analysis on the association between $\mathrm{BMI}$ and breast-ovarian cancer risk found that overweight and obesity could not be considered cancer risk factors in people carrying BRCA1/2 gPV. No increase in cancer risk for people with a BMI exceeding 25 was observed compared to the $<25$ group. Instead, a mild reduction of breast and ovarian cancer risk for the overweight and the obese group has been observed, even if not statistically significant. These surprising results could be explained by the molecular defect determined by BRCA1/2 gPV which results in defective HR, thus affecting genome stability while leaving the cells unable to precisely correct double-strand breaks. Such a condition is likely to be functionally dominant concerning the mutator condition due to overweight/obesity status. Albeit no clear explanation could be drawn, one could speculate that a lower incidence of nutritional deficiencies is present in obeseoverweight and that this effect, masked by the increased rate of mutagenesis observed in the general population, would eventually become visible in BRCA1/2 gPV carriers. Accordingly, in such patients 
mutation rate is driven by the defective BRCA1/2 allele and the contribution of the obesity-dependent mutator phenotype seems to be less important. To our knowledge, no studies have been done on the association between BMI and breast-ovarian cancer risk among the BRCA-carrier population subset. Nonetheless, results need to be interpreted with prudence.

Thus, the present study considered all the literature search results to comprehensively investigate how the BMI status could interplay with the cancer risk in BRCA1/2-positive healthy individuals. Nevertheless, this study had several limitations. First, these results should always be interpreted with caution since they are based on indirect comparisons among only a few studies, and meta-analysis was not performed at the individual-patient level; there was a paucity of data on this subset of research, hence this might limit the study's power. Secondly, a caveat when interpreting results from this study is the small number of included studies; there was also substantial heterogeneity among the studies, which presented a major threat to the validity of the pooled estimates. Thirdly, the literature search matched only case-control study designs, not identifying outcomes of interest among prospective randomized controlled trials and thus possibly restraining our meta-analysis results. Lastly, there was a paucity of data on BRCA 2 mutations in most of the studies involved in our meta-analysis; indeed, only one study treating menopausal status has been finally retrieved in the final analysis.

Although recent progress in the genomic and molecular characterization of breast cancer has led to the identification of new therapeutic targets that may facilitate a more personalized approach in the management of the disease, there is much work to be done in detecting modifiable factors that can shrink the novel breast cancer diagnoses related to the genomic aberration of BRCA $1 / 2$ genes. The roles of body size and physical activity in the etiology of breast cancer among women at baseline population risk have been studied extensively, yet there is a lack of similar data on BRCA-carriers. However, the options currently available for women at increased risk for inherited breast and ovarian cancer consist of surveillance programs aimed at early cancer detection, prophylactic bilateral mastectomy, and prophylactic bilateral adnexectomy, while non-surgical primary prevention options (such as diet, body weight, BMI, low energy intake, physical activity and chemo-preventive options to cease the estrogensignaling pathway) are not yet strongly established. Surely, all these above-mentioned potential risk factors may affect penetrance in BRCA mutation carriers and women belonging to high genetic risk families.

Nowadays, there is a need to be capable to suggest evidence-based recommendations to BRCA-positive women, especially regarding lifestyle choices. Indeed, this is particularly significant in the context of cancer genetic counseling, where the counselor has the power to notably impact a woman's prevention options and lifestyle choices. Finally, our main purpose was to offer novel data that would support the improvement of practical and safe prevention interventions leading to a decrease in the number of breast cancer new cases and deaths attributed to a BRCA mutational status.

\section{Conclusions}


According to our preliminary results, BMI should not be considered as a cancer risk modifier in the BRCA1/2 population. Of course, nonetheless larger studies are necessary to confirm these observations so that evidence-based recommendations are available to assure that BRCA-positive women adopt safe and effective preventive strategies. However, this study suggests caution when drastic lifestyle changes are proposed and encouraged to BRCA1/2 gPV carriers because the relationship between abdominal fat and cancer risk is far to be clear in people with this genetic background. High-quality investigational studies, such as larger prospective and randomized studies, in the BRCA-positive population are thus warranted in order to understand the impact of BMI on the BRCA gene mutations and its role in breast and ovarian cancer incidence and risk in this particular subset.

\section{Abbreviations}

BMI: body mass index

BRCA 1/2: breast cancer susceptibility gene 1 and 2

gPV: pathogenic germline variants

HBOC: hereditary breast-ovarian cancer syndromes

HR : homologous recombination

DSBs: double-strand breaks

BC: breast cancer

OC: ovarian cancer

ASCO: American Society of Clinical Oncology

ESMO: European Society of Medical Oncology

$\mathrm{NIH}$ : National Institute of Health

OR: Odd Ratio

Cl: Confidence Interval

PRE: pre-menopausal Status

POST: post-menopausal Status

NA: not available

PRISMA: Preferred Reporting Items for Systematic Reviews and Meta-Analyzes 


\section{Declarations}

Ethics approval and consent to participate: not applicable

Consent for publication: not applicable

Availability of data and materials: all data generated or analysed during this study are included in this published article

Competing interests: A.R. reports personal fees from Bristol, Pfizer, Bayer, Kyowa Kirin, Ambrosetti for advisory board activity; speaker honorarium from Roche Diagnostics. No other disclosures are reported by the other authors.

Funding: M.G.M. received funding from the Laurus foundation. The remaining authors did not receive any specific fundings for this work.

\section{Authors' contributions}

Conceptualization: M.G.M. and A.G.

Methodology: M.G.M., A.G. and V.G.

Software: A.G., M.LM., M.C. and A.P.

Validation: So.C., St.C., L.I. and L.C.

Formal Analysis: A.G. and V.G.

Investigation: A.G., M.LM. and V.G.

Resources: M.G.M., A.G., A.R. and V.B.

Data Curation: So.C., St.C., V.C., N.B. and M.B.

Writing - Original Draft Preparation: M.G.M., A.G., and V.G.

Writing - Review \& Editing: M.G.M., F.F., A.R. and V.B.

Visualization: V.G., A.R. and V.B.

Supervision: F.F., A.R. and V.B.

Project Administration: A.R. and V.B.

Funding Acquisition: M.G.M. 
Acknowledgements: all the authors thank Dr. Chiara Drago for the English revision contribution. V.G. and M.LM. contributed to the current work under the Doctoral Programme in Experimental Oncology and Surgery, University of Palermo.

\section{References}

1. Paul A, Paul S. The breast cancer susceptibility genes (BRCA) in breast and ovarian cancers. Front Biosci (Landmark Ed). 2014;19:605-18.

2. Shao, D.; Cheng, S.; Guo, F.; Zhu, C.; Yuan, Y.; Hu, K.; Wang, Z.; Meng, X.; Jin, X.; Xiong, Y., et al. Prevalence of hereditary breast and ovarian cancer (HBOC) predisposition gene mutations among 882 HBOC high-risk Chinese individuals. Cancer Sci 2020, 111, 647-657, doi:10.1111/cas. 14242.

3. Angeli D, Salvi S, Tedaldi G. Genetic Predisposition to Breast and Ovarian Cancers: How Many and Which Genes to Test? Int J Mol Sci. 2020;21(3).

4. Nepomuceno TC, De Gregoriis G, de Oliveira FMB, Suarez-Kurtz G, Monteiro AN, Carvalho MA. The Role of PALB2 in the DNA Damage Response and Cancer Predisposition. Int J Mol Sci. 2017;18(9).

5. Somyajit K, Subramanya S, Nagaraju G. RAD51C: a novel cancer susceptibility gene is linked to Fanconi anemia and breast cancer. Carcinogenesis. 2010;31(12):2031-8.

6. Couch FJ, Wang X, McGuffog L, Lee A, Olswold C, Kuchenbaecker KB, et al. Genome-wide association study in BRCA1 mutation carriers identifies novel loci associated with breast and ovarian cancer risk. PLoS Genet. 2013;9(3):e1003212.

7. Mulligan AM, Couch FJ, Barrowdale D, Domchek SM, Eccles D, Nevanlinna H, et al. Common breast cancer susceptibility alleles are associated with tumour subtypes in BRCA1 and BRCA2 mutation carriers: results from the Consortium of Investigators of Modifiers of BRCA1/2. Breast Cancer Res. 2011;13(6):R110.

8. Stevens KN, Wang X, Fredericksen Z, Pankratz VS, Greene MH, Andrulis IL, et al. Evaluation of chromosome $6 \mathrm{p} 22$ as a breast cancer risk modifier locus in a follow-up study of BRCA2 mutation carriers. Breast Cancer Res Treat. 2012;136(1):295-302.

9. Petrucelli N DM, Pal T. BRCA1- and BRCA2-Associated Hereditary Breast and Ovarian Cancer. In: Adam MP, Ardinger HH, Pagon RA, Wallace SE, Bean LJH, Stephens K, et al., editors. GeneReviews(®). Seattle (WA): University of Washington, Seattle Copyright $($ 1993-2020, University of Washington, Seattle. GeneReviews is a registered trademark of the University of Washington, Seattle. All rights reserved., 1993.

10. Kotsopoulos J. Mutations and Breast Cancer Prevention. Cancers (Basel). 2018;10(12).

11. Sun L, Brentnall A, Patel S, Buist DSM, Bowles EJA, Evans DGR, et al. A Cost-effectiveness Analysis of Multigene Testing for All Patients With Breast Cancer. JAMA Oncology. 2019;5(12):1718-30.

12. Pilarski R. The Role of BRCA Testing in Hereditary Pancreatic and Prostate Cancer Families. American Society of Clinical Oncology Educational Book. 2019(39):79-86.

13. Wolin KY, Carson K, Colditz GA. Obesity and cancer. Oncologist. 2010;15(6):556-65. 
14. Reeves GK, Pirie K, Beral V, Green J, Spencer E, Bull D, et al. Cancer incidence and mortality in relation to body mass index in the Million Women Study: cohort study. BMJ. 2007;335(7630):1134.

15. Calle EE, Rodriguez C, Walker-Thurmond K, Thun MJ. Overweight, obesity, and mortality from cancer in a prospectively studied cohort of U.S. adults. N Engl J Med. 2003;348(17):1625-38.

16. Juge-Aubry CE, Henrichot E, Meier CA. Adipose tissue: a regulator of inflammation. Best Pract Res Clin Endocrinol Metab. 2005;19(4):547-66.

17. Renehan AG, Tyson M, Egger M, Heller RF, Zwahlen M. Body-mass index and incidence of cancer: a systematic review and meta-analysis of prospective observational studies. Lancet. 2008;371(9612):569-78.

18. Wang J, Yang DL, Chen ZZ, Gou BF. Associations of body mass index with cancer incidence among populations, genders, and menopausal status: A systematic review and meta-analysis. Cancer Epidemiol. 2016;42:1-8.

19. Key TJ, Allen NE, Spencer EA, Travis RC. The effect of diet on risk of cancer. Lancet. 2002;360(9336):861-8.

20. Kaaks R, Rinaldi S, Key TJ, Berrino F, Peeters PH, Biessy C, et al. Postmenopausal serum androgens, oestrogens and breast cancer risk: the European prospective investigation into cancer and nutrition. Endocr Relat Cancer. 2005;12(4):1071-82.

21. Macis D, Guerrieri-Gonzaga A, Gandini S. Circulating adiponectin and breast cancer risk: a systematic review and meta-analysis. Int J Epidemiol. 2014;43(4):1226-36.

22. Rangel-Huerta OD, Pastor-Villaescusa B, Gil A. Are we close to defining a metabolomic signature of human obesity? A systematic review of metabolomics studies. Metabolomics. 2019;15(6):93.

23. Basen-Engquist $K$, Chang M. Obesity and cancer risk: recent review and evidence. Curr Oncol Rep. 2011;13(1):71-6.

24. Strulov Shachar S, Williams GR. The Obesity Paradox in Cancer-Moving Beyond BMI. Cancer Epidemiol Biomarkers Prev. 2017;26(1):13-6.

25. Fu Y, Zhuang Z, Dewing M, Apple S, Chang H. Predictors for contralateral prophylactic mastectomy in breast cancer patients. Int J Clin Exp Pathol. 2015;8(4):3748-64.

26. Bissonauth V, Shatenstein B, Fafard E, Maugard C, Robidoux A, Narod S, et al. Weight History, Smoking, Physical Activity and Breast Cancer Risk among French-Canadian Women Non-Carriers of More Frequent BRCA1/2 Mutations. J Cancer Epidemiol. 2009;2009:748367.

27. Khachatryan L, Scharpf R, Kagan S. Influence of diabetes mellitus type 2 and prolonged estrogen exposure on risk of breast cancer among women in Armenia. Health Care Women Int. 2011;32(11):953-71.

28. Nkondjock A, Robidoux A, Paredes Y, Narod SA, Ghadirian P. Diet, lifestyle and BRCA-related breast cancer risk among French-Canadians. Breast Cancer Res Treat. 2006;98(3):285-94.

29. Wells G SB, O'Connell D, Peterson J, Welch V, Losos M, Tugwell P. The Newcastle-Ottawa Scale (NOS) for assessing the quality of nonrandomised studies in meta-analyses. 2013. 
30. Comprehensive Meta-Analysis Version 3 Borenstein M, Hedges, L., Higgins, J., \& Rothstein, H. Biostat, Englewood, NJ 2013.

31. Borenstein M, Hedges LV, Higgins JP, Rothstein HR. A basic introduction to fixed-effect and randomeffects models for meta-analysis. Res Synth Methods. 2010;1(2):97-111.

32. Moher D, Liberati A, Tetzlaff J, Altman DG, Group P. Preferred reporting items for systematic reviews and meta-analyses: the PRISMA statement. PLoS Med. 2009;6(7):e1000097.

33. Vucenik I, Stains JP. Obesity and cancer risk: evidence, mechanisms, and recommendations. Ann N Y Acad Sci. 2012;1271:37-43.

34. Niraula S, Ocana A, Ennis M, Goodwin PJ. Body size and breast cancer prognosis in relation to hormone receptor and menopausal status: a meta-analysis. Breast Cancer Res Treat. 2012;134(2):769-81.

35. Xia X, Chen W, Li J, Chen X, Rui R, Liu C, et al. Body mass index and risk of breast cancer: a nonlinear dose-response meta-analysis of prospective studies. Sci Rep. 2014;4:7480.

36. Mirisola MG, Taormina G, Fabrizio P, Wei M, Hu J, Longo VD. Serine- and threonine/valine-dependent activation of PDK and Tor orthologs converge on Sch9 to promote aging. PLoS Genet. 2014;10(2):e1004113.

\section{Supplementary Files}

This is a list of supplementary files associated with this preprint. Click to download.

- FigureSupplementary1.docx

- FigureSupplementary2.docx 